\title{
NUMERICAL SIMULATION OF BALLAST WATER BY SPH METHOD
}

\author{
YANG XIUFENG*, PENG SHILIU ${ }^{\dagger}$, LIU MOUBIN ${ }^{\ddagger}$ \\ and SHAO JIARU ${ }^{\S}$ \\ Institute of Mechanics, Chinese Academy of Sciences \\ Beisihuanxi Road, Beijing, China \\ *yangxf@imech.ac.cn \\ †slpeng@imech.ac.cn \\ ¥liumoubin@imech.ac.cn \\ §shaojiaru@163.com \\ Received 18 October 2010 \\ Accepted 2 May 2011
}

\begin{abstract}
Ballast water has frequently been used in ships to provide stability and adjust trim, stress, and torsion for optimal steering and propulsion. Numerical simulation of the movement of ballast water and its interaction with the solid walls of operating ships are very difficult for traditional grid-based numerical models. In this paper, the smoothed particle hydrodynamics (SPH) method is applied to simulate water tank sloshing and the movement of ships carrying ballast water in three cases. Numerical results of water tank sloshing are compared with experimental ones. Numerical results of ships indicate that carrying ballast water in several separated small tanks generally makes a ship more stable, but keeping ballast water in one big tank generally makes a ship more unstable.
\end{abstract}

Keywords: Ballast water; smoothed particle hydrodynamics; sloshing; anti-roll tank.

\section{Introduction}

A ship must be a stable platform, whether at rest or moving under the influence of wind, currents, and waves. For a period of long time, people are trying to stabilize ships. It is usually taken into ballast tanks when cargo is being offloaded, and discharged when cargo is being loaded. Ships have carried solid ballast for thousands of years. In modern times, ships use water as ballast. The tanks on ships wherein the ballast water is filled are known as ballast tanks or anti-roll tanks. The ballast tanks are located at the lowermost region of a ship. The movement of the free surface of ballast water is a typical phenomenon of sloshing. The rolling movement of a ship can be modeled as a one-degree harmonic movement. The ballast water follows the ship's rolling and acquires a phase lag with respect to the ship movement. If this lag is $90^{\circ}$, the moment generated by the ballast water at its maximum can partially counteract the sea wave moment outside the ship.

\footnotetext{
${ }^{\ddagger}$ Corresponding author.
} 
Numerical simulation of the movement of ballast water is very difficult for traditional grid-based numerical models, as it involves not only complex geometries and free surfaces, but also fluid-solid interactions as well as other complex physics in a comparably very large scale. In many circumstances, violent fluid-structure interactions lead to breaking water, air entrapment, and multi-phase flows, where the dynamics of the entrapped air at the impact may play a dominant role during the process and contribute to the high-pressure maxima and pressure oscillations. Though conventional grid-based methods such as the finite difference method (FDM), the finite volume method (FVM), and the finite element method (FEM) have achieved greatly in simulating fluid flow in ocean hydrodynamics and coastal engineering, there is still a long way to go for practical engineering applications in modeling the movement of ballast water and its interaction with surrounding ship cabins.

Smoothed particle hydrodynamics (SPH) is a fully meshfree Lagrangian particle method and it has been successfully applied to a wide range of problems. In $\mathrm{SPH}$ method, the equations of fluid dynamics are solved numerically by replacing the fluid with a set of particles. The SPH method was originally proposed to deal with astrophysics problems [Gingold and Monaghan (1977); Lucy (1977)]. Later, it was used for a wide range of fluid dynamics problems. By incorporating an artificial viscosity, SPH method can be applied to one-dimensional (1D) shock tube problems [Monaghan and Gingold (1983)]. Liu et al. [2003] simulated the underwater explosion problem by making some modifications in SPH code. Lo and Shao [2002] simulated the near-shore solitary wave mechanics by an incompressible SPH method together with a large eddy simulation approach. Hu and Adams [2007] proposed an incompressible multi-phase SPH method. Yang and Peng [2010] simulated dam break flow by SPH method with a new kernel function. More details of the $\mathrm{SPH}$ method and its recent developments can be found in a number of review papers [Cleary et al. (2007); Liu and Liu (2010); Monaghan (2005)].

Simulation of sloshing problems using SPH is a promising research direction. Souto-Iglesias et al. [2004] simulated the anti-roll tanks and sloshing type problems. Delorme et al. [2009] investigated experimentally and numerically impact pressure in the case of shallow water sloshing for forced-rolling motion. Rhee [2005] studied liquid tank sloshing with Reynolds-averaged Navier-Stokes. Souto-Iglesias et al. [2006] assessed the liquid moment amplitude in sloshing type problems with smooth particle hydrodynamics. Bulian et al. [2010] simulated a tuned liquid damper with angular motion. Anghileri et al. [2005] investigated the fluid-structure interaction of water-filled tanks during the impact with the ground. Delorme [2008] experimentally investigated and numerically simulated with SPH the sloshing flows in a anti-roll tank and LNG tankers.

In this paper, a weakly compressible SPH method will be used to simulate water sloshing in a rectangular tank and the movement of ships carrying ballast water in three cases. 


\section{SPH Equations}

\subsection{SPH methodology}

In the SPH method, for any function $f(\boldsymbol{r})$ that is a function of the spatial coordinates, the value of function $f$ at a certain point $a$ whose position vector is $\boldsymbol{r}_{a}$ can be approximated by the following integral interpolation:

$$
f\left(\boldsymbol{r}_{a}\right)=\int_{\Omega} f(\boldsymbol{r}) W\left(\boldsymbol{r}_{a}-\boldsymbol{r}, h\right) \mathrm{d} V,
$$

where $W\left(\boldsymbol{r}_{a}-\boldsymbol{r}, h\right)$ is a kernel function, $h$ is a smoothing length, $\mathrm{d} V$ is a differential volume element, and $\Omega$ is an integral domain. The interpolation reproduces $f$ exactly if the kernel is a delta function. In practice, the value of $h$ determines the range of the integral domain and the kernel $W$ tends to the delta function as the smoothing length $h$ tends to zero. Kernels are normalized to 1 , so that constants are interpolated exactly.

A commonly used kernel is the cubic spline function:

$$
M_{4}(s, h)=\alpha_{d} \begin{cases}(2-s)^{3}-4(1-s)^{3}, & 0 \leq s<1 \\ (2-s)^{3}, & 1 \leq s<2 \\ 0, & s \geq 2\end{cases}
$$

where $s=|\boldsymbol{r}| / h$, and $\alpha_{d}$ is $1 /(6 h), 15 /\left(14 \pi h^{2}\right)$, and $1 /\left(4 \pi h^{3}\right)$ in $1 \mathrm{D}, 2 \mathrm{D}$, and $3 \mathrm{D}$ spaces, respectively.

To apply Eq. (1) to fluid, the interpolation is approximated by a summation interpolation over a set of small particles:

$$
f_{a}=\sum_{b} f_{b} W_{a b} \frac{m_{b}}{\rho_{b}},
$$

where $W_{a b}=W\left(\boldsymbol{r}_{a}-\boldsymbol{r}_{b}, h\right)$, the indexes $a$ and $b$ denote labels of particles, e.g. $f_{a}$ denotes the value of quantity $f$ at $\boldsymbol{r}_{a}$ and particle $b$ has position $\boldsymbol{r}_{b}$, mass $m_{b}$, density $\rho_{b}$, and volume $m_{b} / \rho_{b}$. The summation is over all particles but in practice it is only over near neighbors because the kernel $W$ vanishes outside its support domain (Fig. 1).

The gradient of $f$ can be obtained by differentiating Eq. (3) exactly:

$$
\nabla f_{a}=\sum_{b} \frac{m_{b}}{\rho_{b}} f_{b} \nabla_{a} W_{a b}
$$

where $\nabla_{a} W_{a b}=W_{a b}^{\prime} \boldsymbol{r}_{a b} / \boldsymbol{r}_{a b}$ is the gradient of the kernel taken with respect to the position of particle $a$. This form of the gradient does not vanish if $f$ is a constant. Using a few mathematical techniques, the gradient can be expressed as:

$$
(\nabla f)_{a}=\sum_{b} \frac{m_{b}}{\rho_{b}}\left(f_{b}-f_{a}\right) \nabla_{a} W_{a b} .
$$

The gradient vanishes if $f$ is a constant. 


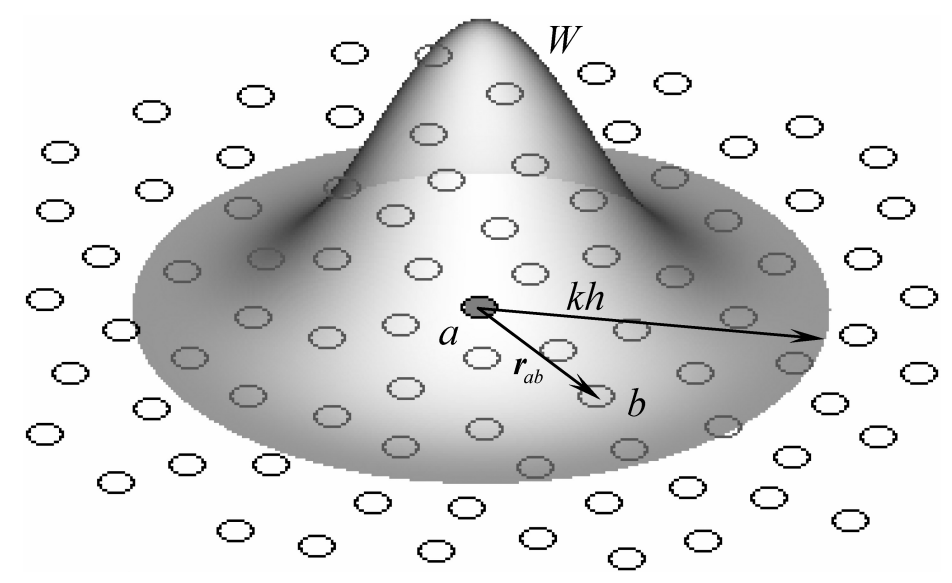

Fig. 1. Kernel interpolation of particles.

\subsection{Governing equations for fluid}

The Lagrangian form of the Navier-Stokes equations are written as follows:

$$
\begin{aligned}
\frac{\mathrm{d} \rho}{\mathrm{d} t} & =-\rho \nabla \cdot \boldsymbol{u}, \\
\frac{\mathrm{d} \boldsymbol{u}}{\mathrm{d} t} & =\boldsymbol{g}-\frac{1}{\rho} \nabla p+\frac{\mu}{\rho} \nabla^{2} \boldsymbol{u},
\end{aligned}
$$

where $\rho$ is fluid density, $\boldsymbol{u}$ is the velocity vector, $p$ is pressure, $\mu$ is the dynamic viscosity, and $\boldsymbol{g}$ denotes the body force including the gravitational acceleration.

In $\mathrm{SPH}$, the Navier-Stokes equations can be expressed as:

$$
\begin{aligned}
\frac{\mathrm{d} \rho_{a}}{\mathrm{~d} t} & =\sum_{b} m_{b} \boldsymbol{u}_{a b} \cdot \nabla_{a} W_{a b}, \\
\frac{\mathrm{d} \boldsymbol{u}_{a}}{\mathrm{~d} t} & =\boldsymbol{g}_{a}-\sum_{b} m_{b}\left(\frac{P_{a}}{\rho_{a}^{2}}+\frac{P_{b}}{\rho_{b}^{2}}\right) \cdot \nabla_{a} W_{a b}+\sum_{b} \frac{m_{b}\left(\mu_{a}+\mu_{b}\right) \boldsymbol{r}_{a b} \cdot \nabla_{a} W_{a b}}{\rho_{a} \rho_{b}\left(r_{a b}^{2}+0.01 h^{2}\right)} \boldsymbol{u}_{a b},
\end{aligned}
$$

where $\boldsymbol{u}_{a b}=\boldsymbol{u}_{a}-\boldsymbol{u}_{b}$.

In most SPH literature, incompressible liquids such as water are usually considered to be slight compressible, using a weakly compressible concept, which satisfies an artificial equation of state [Morris et al. (1997)]

$$
P(\rho)=c^{2}\left(\rho-\rho_{0}\right),
$$

where $\rho_{0}$ is a reference density and $c$ is a numerical speed of sound. In order to reduce the density fluctuation down to one percent, $c$ is usually taken 10 times higher than the maximum fluid velocity. 


\subsection{Governing equations for rigid body}

For a rigid body, the equation for the motion of the center of mass can be written as follows:

$$
\frac{\mathrm{d} \boldsymbol{u}_{o}}{\mathrm{~d} t}=\frac{\boldsymbol{F}}{M}+\boldsymbol{g}
$$

where $\boldsymbol{u}_{o}$ is the velocity of the center of mass, $\boldsymbol{F}$ is the summation of forces without gravity around the rigid body, and $M$ is the mass of the rigid body.

In the case of $2 \mathrm{D}$ motion, the equation for the angular velocity is

$$
\frac{\mathrm{d} \omega}{\mathrm{d} t}=\frac{J}{I}
$$

where $\omega$ is the angular velocity, $I$ is the moment of inertia, and $\boldsymbol{J}$ is the total moment of force on the rigid body about the center of mass.

The velocity of a point $a$ on the rigid body is

$$
\boldsymbol{u}_{a}=\boldsymbol{u}_{o}+\omega \times \boldsymbol{r}_{o a},
$$

where $\boldsymbol{r}_{o a}$ is the vector from the center of mass $o$ to point $a$.

In SPH method, $\boldsymbol{F}$ and $\boldsymbol{J}$ can be expressed as follows:

$$
\begin{aligned}
& \boldsymbol{F}=\sum_{b} \boldsymbol{f}_{b}, \\
& \boldsymbol{J}=\sum_{b} \boldsymbol{r}_{o b} \times \boldsymbol{f}_{b},
\end{aligned}
$$

where $\boldsymbol{f}_{b}$ denotes the force on particle $b$ due to fluid. The summation is overall rigid body particles.

\section{Numerical Examples}

A free ship has six degrees of freedom (DOF) (surge, sway, heave, roll, pitch, and yaw, see Fig. 2). Out of the six motions, the critical motion leading to capsize of a

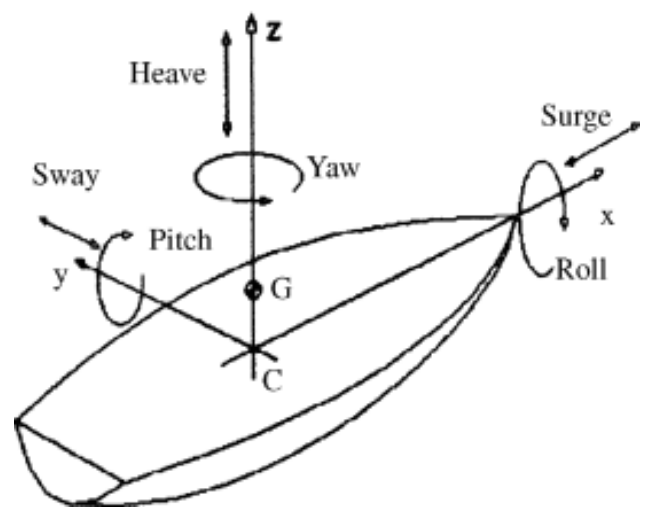

Fig. 2. Coordinate axis and definition of the six degrees of motions [Neves and Rodriguez (2006)]. 
ship is the rolling motion. Here, it is reasonable to investigate the ship motion as a 2D problem in $C y z$ plane.

\subsection{Sloshing}

In this section, the rolling motion is studied by simulating sloshing in a partially filled tank. Anti-roll tank problem has been experimentally investigated and numerically simulated by many people. In order to test the numerical method and compare with experimental results, the tank configure is the same as used in the paper SoutoIglesias et al. [2006]. As showing in Fig. 3, the tank has a length of $64 \mathrm{~cm}$, a height of $14 \mathrm{~cm}$, and a width of $25.2 \mathrm{~cm}$. The tank is partially filled with water of $3 \mathrm{~cm}$ depth. The rotation axis is $10 \mathrm{~cm}$ below its baseline. The rotational angle of the tank in degrees is $\theta=6 \sin (\omega t)$. The initial space between particles is $0.2 \mathrm{~cm}$, and 4,800 fluid particles are used in the simulation.

The moment of momentum $M_{t}$ is calculated as follows:

$$
M_{t}=\sum_{b} \boldsymbol{f}_{b} \times \boldsymbol{r}_{o b}
$$

where $\boldsymbol{r}_{o b}$ denotes the vector from rolling center $o$ to point $b$. The summation is overall boundary particles that represent the tank walls.

In order to obtain a harmonic whose frequency is equal to the excitation frequency of the tank, the numerical moment is filtered as follows:

$$
M_{1}=A_{1} \sin \left(\omega t-\alpha_{1}\right),
$$

where $A_{1}$ is the moment amplitude and $\alpha_{1}$ is phase lag. Simulation is applied to five excitation periods, but filtering ignores approximately the first period at the beginning. Filtered moment for $\omega=1.0 \mathrm{rad} / \mathrm{s}$ is shown in Fig. 4. The filtered and nonfiltered moments agree with each other very well. However, for high excitation frequencies, filtered moments using Eq. (17) do not agree with nonfiltered moments so well (Fig. 5).

In order to obtain good filtered moments for high frequencies, a combinational function of two harmonics with different frequencies as follows is used:

$$
M_{2}=A_{2} \sin \left(\omega t-\alpha_{2}\right)+B \sin (\varpi t-\beta),
$$

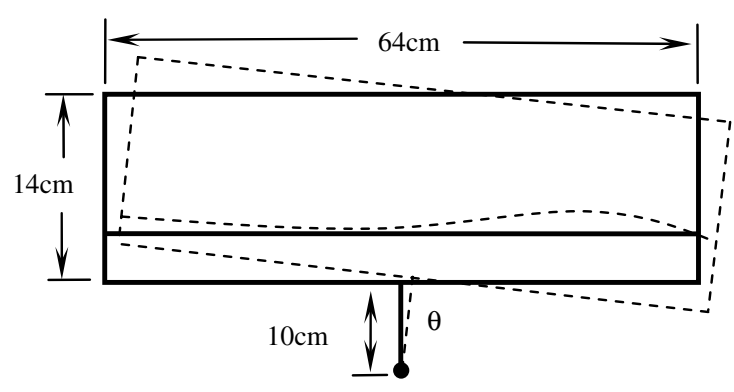

Fig. 3. Anti-roll tank sketch. 


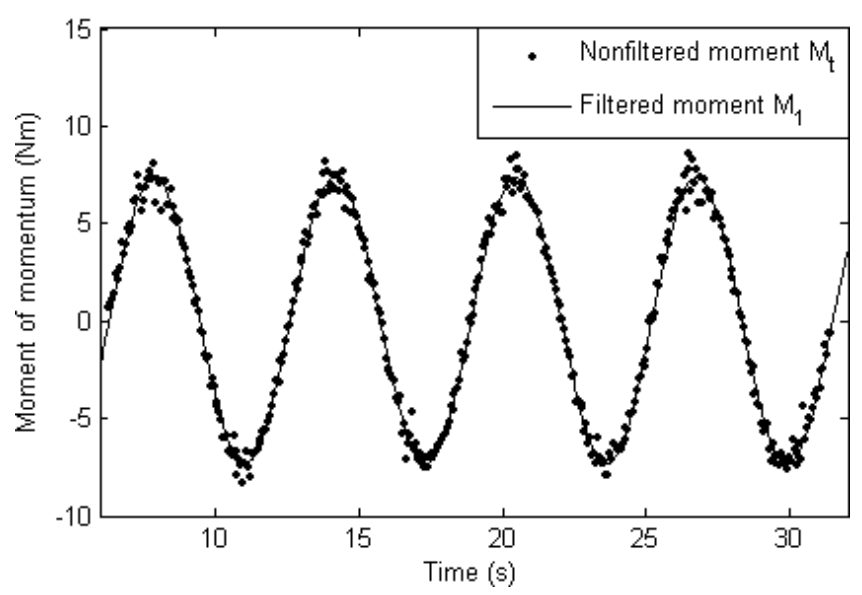

Fig. 4. Filtered moment for $\omega=1.0 \mathrm{rad} / \mathrm{s}$.

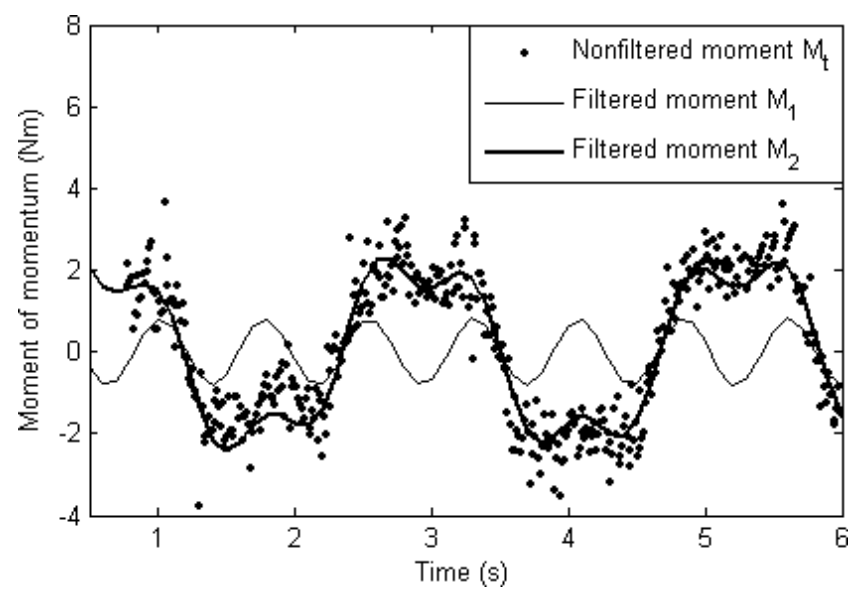

Fig. 5. Filtered moment for $\omega=8.21 \mathrm{rad} / \mathrm{s}$.

where $A_{2}$ is the moment amplitude and $\alpha_{2}$ is the phase lag corresponding to excitation frequency $\omega . B$ is a second amplitude corresponding to a second frequency $\varpi$, which do not equal to $\omega$, and $\beta$ is a second phase lag. Filtered moment for $\omega=8.21 \mathrm{rad} / \mathrm{s}$ is shown in Fig. 5 . We can see that filtered moment $M_{2}$ agrees with nonfiltered moment much better than $M_{1}$.

Figure 6 shows the comparison of simulation values of the phase lags and experimental results. The simulation results for low frequencies agree with experimental ones very well, but not so well for high frequencies.

Figure 7 is the moment amplitudes for different frequencies. The simulation results agree with experimental ones very well for most frequencies, except for frequencies around $5 \mathrm{rad} / \mathrm{s}$. For frequencies bigger than $6.5 \mathrm{rad} / \mathrm{s}$, the second 


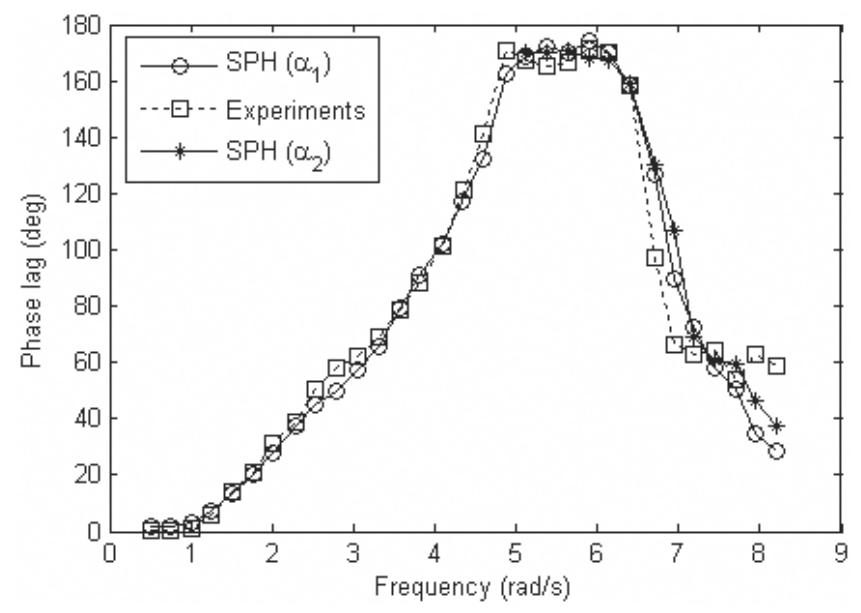

Fig. 6. Comparison of phase lag between SPH and experiments.

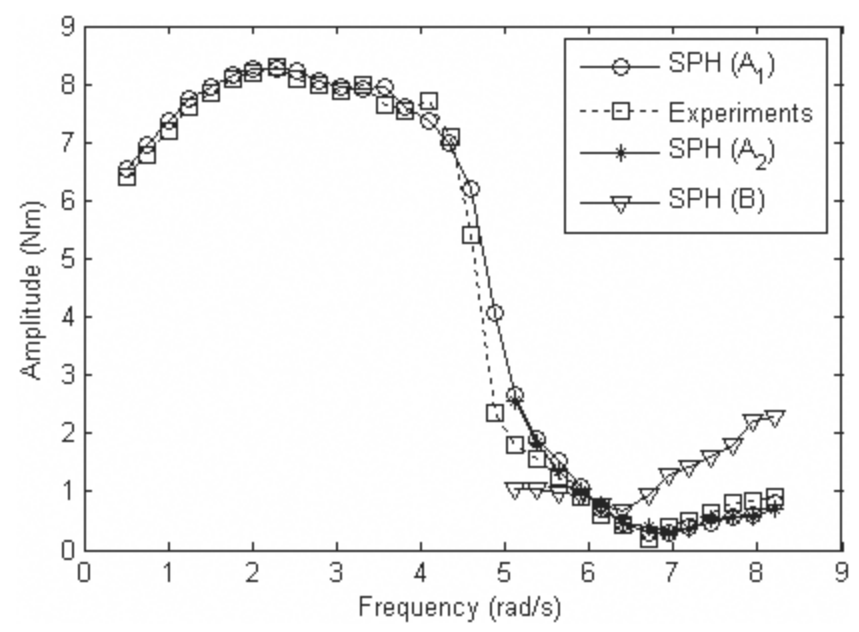

Fig. 7. Comparison of moment amplitude between SPH and experiments.

amplitudes are much bigger than the amplitudes corresponding to excitation frequencies. The second frequencies for high excitation frequencies are shown in Fig. 8.

\subsection{Ballast water}

The rolling motion coupling with the motions of heave and sway of a ship with partially filled tanks is investigated in this section. There are many types of ballast tanks. Rectangular tanks without any control system are used here. A sketch of a $2 \mathrm{D}$ ship carrying ballast water in a wave tank is shown in Fig. 10. A wave maker undergoes simple harmonic motion to produce water waves. The amplitude of the 


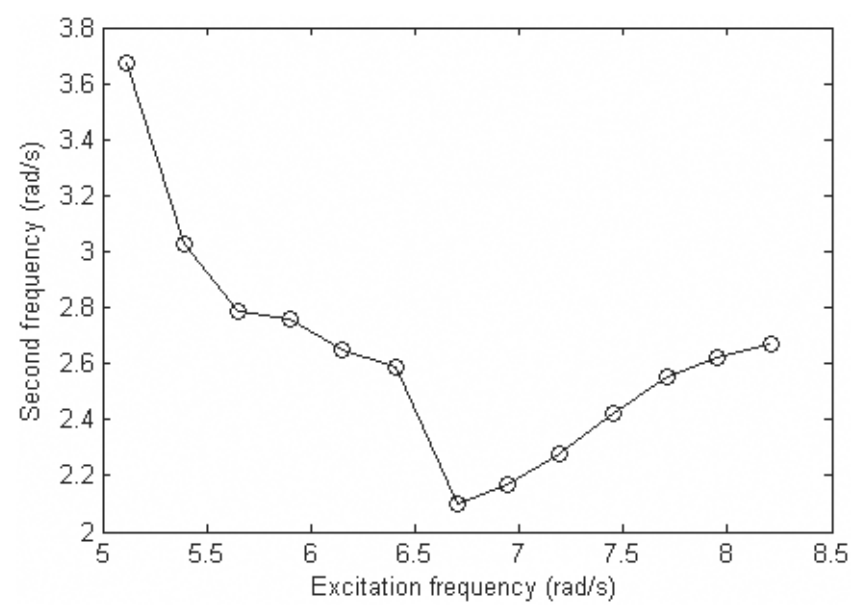

Fig. 8. Excitation frequency versus second frequency.

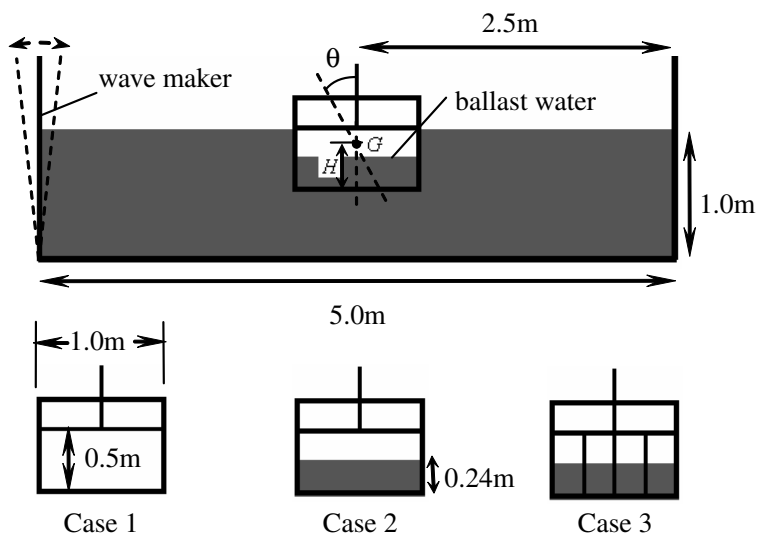

Fig. 9. Sketch of the computational domain and three cases of ballast water simulations (Case 1: no ballast water; Case 2: carrying ballast water in a single big tank; and Case 3: carrying ballast water in four small tanks).

harmonic motion is $2.5^{\circ}$ and the period is $1 \mathrm{~s}$. The net weight of the ship is $250 \mathrm{~kg}$, the moment of inertia is $32.55 \mathrm{~kg} \cdot \mathrm{m}^{2}$, and the wall thickness is $0.04 \mathrm{~m}$. $G$ is the center of gravity of the ship without ballast water, $H$ is $G$ 's height from the bottom of the ship, and $\theta$ is the roll angle. We investigate the stability of the ship by carrying ballast water in three different cases (Fig. 9) and changing the value of $H$. The initial space is $0.01 \mathrm{~m}$, and about 47,500 fluid particles are used.

Figure 10 compares the roll angles between three cases with different $H$ values. For $H=0.25 \mathrm{~m}$, the roll angles approximately satisfy harmonic motion. The angle amplitudes of Cases 2 and 3 are bigger than that of Case 1, which means ballast 

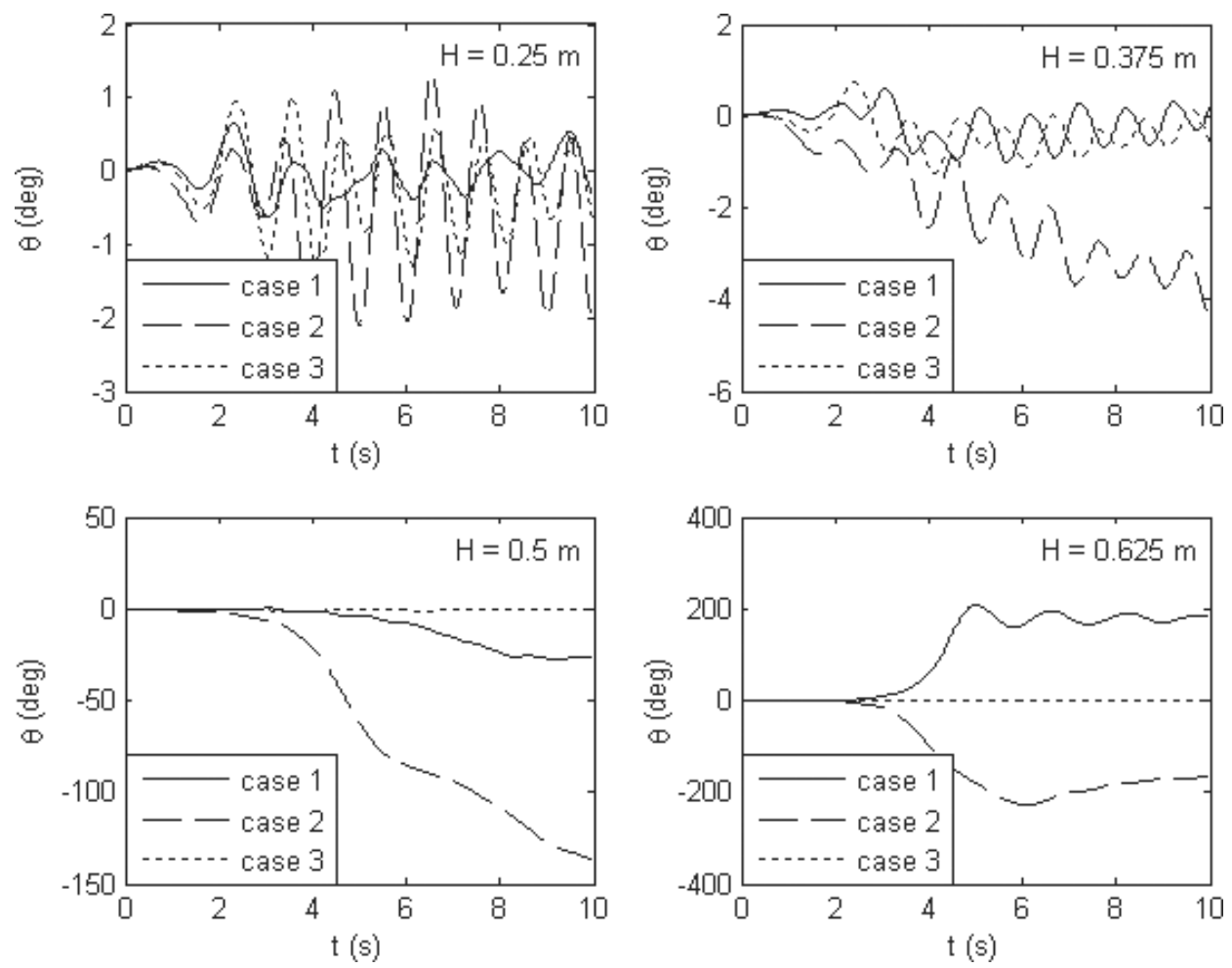

Fig. 10. Comparisons of roll angles between three cases with different $H$ values.

water increases roll angle but not too much. For $H=0.375 \mathrm{~m}$, the roll angles of Case 2 are much bigger than that of Cases 1 and 3 . For $H=0.5 \mathrm{~cm}$, roll angles of Case 2 are much bigger than that of Case 1, and bigger than $90^{\circ}$ for $t>6 \mathrm{~s}$, which means the ship capsizes. The roll angles of Case 3 can be ignored comparing with Case 1; this means that carrying ballast water in separated tanks makes the ship more stable. For $H=0.625 \mathrm{~m}$, ships in Cases 1 and 3 both capsize, but the ship in Case 2 is still very stable.

Figure 11 shows the comparison of the configurations of Cases 2 and 3 with $H=0.625 \mathrm{~m}$. For Case 2, we can see that when the ship carrying ballast water in one big tank tilts to the right side, the ballast water tends to shift toward the right side, too. Therefore, the center of gravity of the ballast water shifts. Instead of righting the ship, the ballast water in the ship turns the ship in the same direction of tilt, and the ship rotates and capsizes. This phenomenon is called free surface effect. For Case 3, the ship carrying ballast water in four small tanks does not capsize, because although the center of gravity of individual ballast water shift, but the summation of all the center of gravities does not shift the center of the ship that significantly as the ballast water in one big tank. 


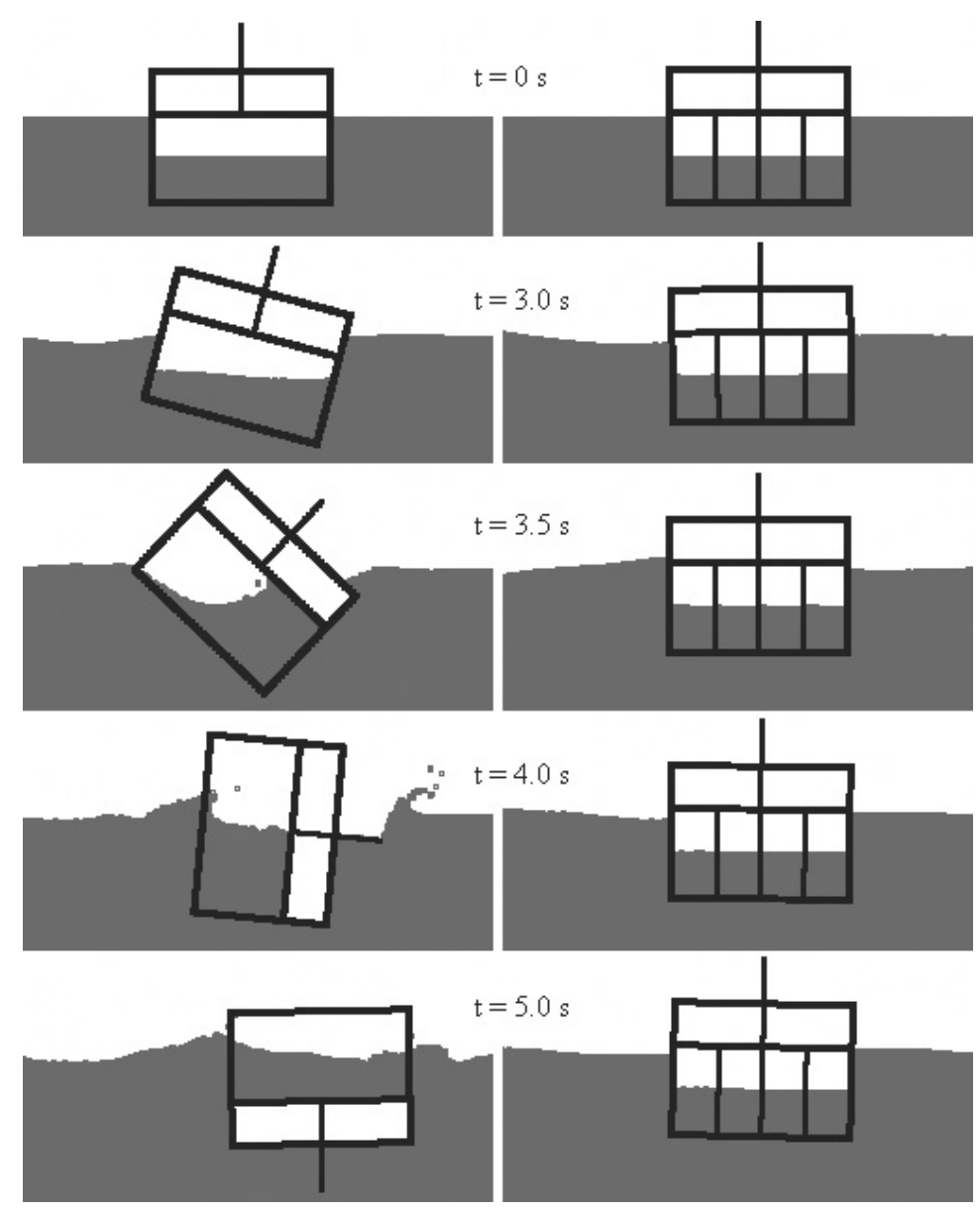

Fig. 11. Comparison of the configurations of Cases 2 (left) and 3 (right) with $H=0.625 \mathrm{~m}$.

\section{Conclusions}

The SPH method is applied to simulate sloshing and ballast water problems. It is capable of simulating the whole process of ship rolling and capsizing. The SPH and experimental results agree with each other very well in sloshing problems. For ballast water problem, in the general case, a ship carrying ballast water in several tanks is more stable than carrying no ballast water, but a ship carrying ballast water in only one big tank is more unstable than carrying nothing.

\section{References}

Anghileri, M., Castelletti, L. M. L., and Tirelli, M. [2005] Fluid-structure interaction of water filled tanks during the impact with the ground, Int. J. Impact Eng. 31, 235-254. 
Bulian, G., Souto-Iglesias, A., Delorme, L., and Botia-Vera, E. [2010] Smoothed particle hydrodynamics (SPH) simulation of a tuned liquid damper (TLD) with angular motion, J. Hydraul. Res. 48, 28-39.

Cleary, P. W., Prakash, M., Ha, J., and Stokes, N. [2007] Smooth particle hydrodynamics: status and future potential, Prog. Comput. Fluid Dyn. Int. J. 7, 70-90.

Delorme, L. [2008] Sloshing flows. Experimental investigation and numerical simulations with smoothed particle hydrodynamics, Ph. D. Thesis, Universidad Politecnica de Madrid.

Delorme, L., Colagrossi, A., Souto-Iglesias, A., Zamora-Rodriguez, R., and Botia-Vera, E. [2009] A set of canonical problems in sloshing, Part I: Pressure field in forced roll comparison between experimental results and SPH, Ocean Eng. 36, 168-178.

Gingold, R. A. and Monaghan, J. J. [1977] Smoothed particle hydrodynamics: theory and application to non-spherical stars, Mon. Not. R. Astron. Soc. 181, 375-389.

Hu, X. and Adams, N. [2007] An incompressible multi-phase SPH method, J. Comput. Phys. 227, 264-278.

Liu, M. B. and Liu, G. R. [2010] Smoothed Particle Hydrodynamics (SPH): an Overview and Recent Developments, Arch. Comput. Methods Eng. 17, 25-76.

Liu, M. B., Liu, G. R., Lam, K. Y., and Zong, Z. [2003] Smoothed particle hydrodynamics for numerical simulation of underwater explosion, Comput. Mech. 30, 106-118.

Lo, Y. M. E. and Shao, S. D. [2002] Simulation of near-shore solitary wave mechanics by an incompressible SPH method, Appl. Ocean Res. 24, 275-286.

Lucy, L. B. [1977] A numerical approach to the testing of the fission hypothesis, The Astron. J. 82, 1013-1024.

Monaghan, J. J. [2005] Smoothed particle hydrodynamics, Rep. Prog. Phys. 68, 1703-1759.

Monaghan, J. J. and Gingold, R. A. [1983] Shock simulation by the particle method SPH, J. Comput. Phy. 52, 374-389.

Morris, J. P., Fox, P. J., and Zhu, Y. [1997] Modeling low reynolds number incompressible flows using SPH, J. Comput. Phy. 136, 214-226.

Neves, M. A. S. and Rodriguez, C. A. [2006] On unstable ship motions resulting from strong non-linear coupling, Ocean Eng. 33, 1853-1883.

Rhee, S. H. [2005] Unstructured grid based Reynolds-Averaged Navier-Stokes method for liquid tank sloshing, J. Fluids Eng. 127, 572.

Souto-Iglesias, A., Delorme, L., Perez-Rojas, L., and Abril-Perez, S. [2006] Liquid moment amplitude assessment in sloshing type problems with smooth particle hydrodynamics, Ocean Eng. 33, 1462-1484.

Souto-Iglesias, A., Perez-Rojas, L., and Zamora-Rodriguez, R. [2004] Simulation of antiroll tanks and sloshing type problems with smoothed particle hydrodynamics, Ocean Eng. 31, 1169-1192.

Yang, X. F. and Peng, S. L. [2010] Simulation of dam-break flow with SPH method, Chinese J. Comput. Phy. 27, 173-180. 